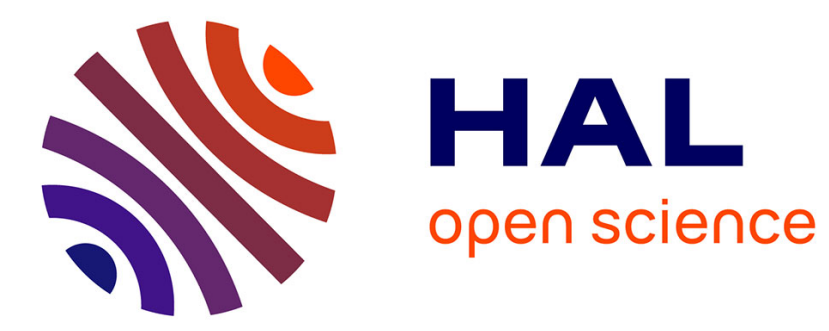

\title{
Bouger pour grandir. Défection et mobilité des adolescents
}

Joël Zaffran

\section{To cite this version:}

Joël Zaffran. Bouger pour grandir. Défection et mobilité des adolescents. Les Annales de la Recherche Urbaine, 2016, 111, pp.69-77. halshs-01309308

\section{HAL Id: halshs-01309308 \\ https://shs.hal.science/halshs-01309308}

Submitted on 29 Apr 2016

HAL is a multi-disciplinary open access archive for the deposit and dissemination of scientific research documents, whether they are published or not. The documents may come from teaching and research institutions in France or abroad, or from public or private research centers.
L'archive ouverte pluridisciplinaire HAL, est destinée au dépôt et à la diffusion de documents scientifiques de niveau recherche, publiés ou non, émanant des établissements d'enseignement et de recherche français ou étrangers, des laboratoires publics ou privés. 


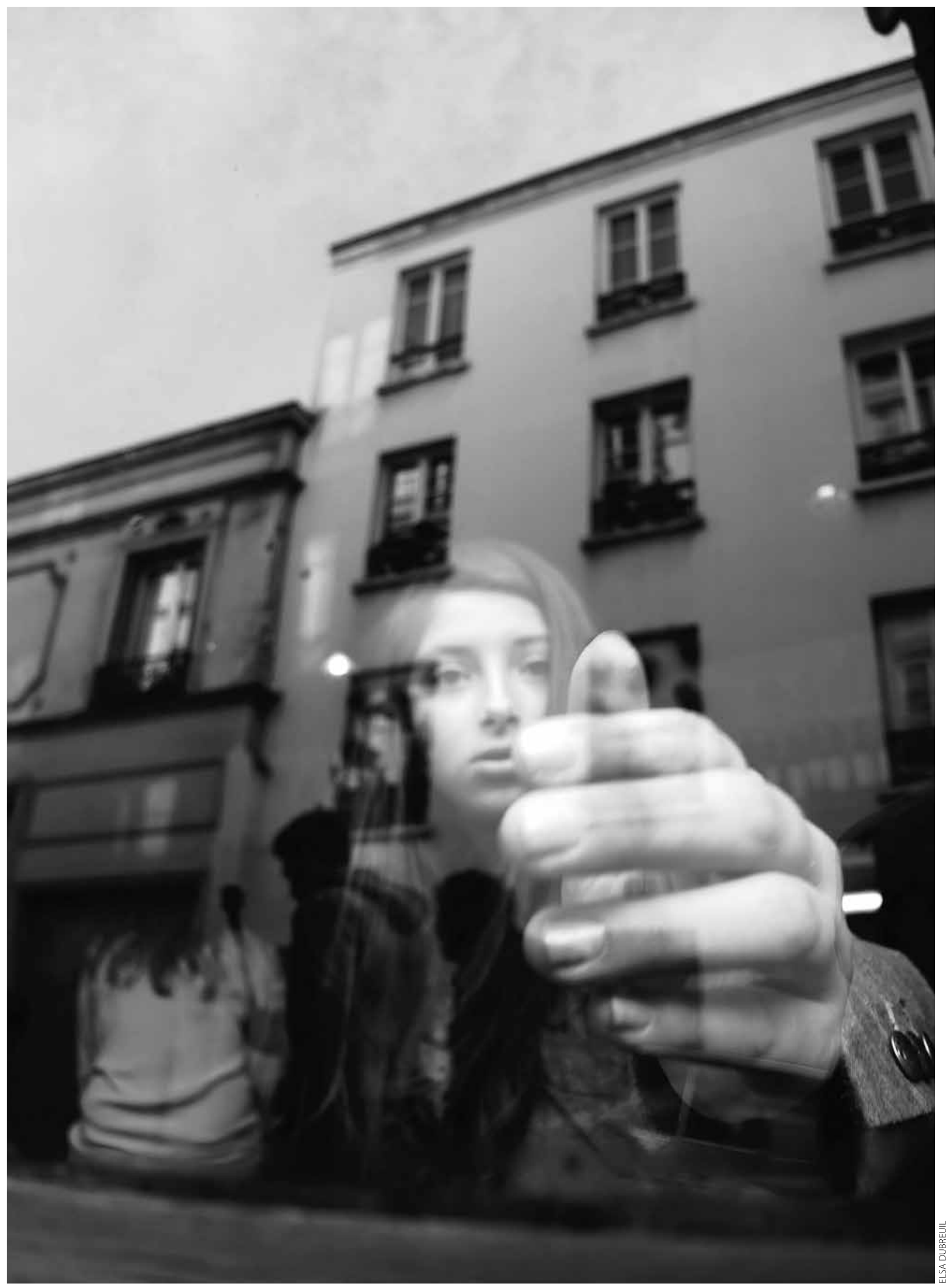




\section{Bouger pour grandir}

Défection et mobilité des adolescents

\section{Joël Zaffran}

Le rapport des adolescents à la ville est marqué par une défection des équipements urbains mis à leur disposition. Une évaluation de la Caisse nationale des allocations familiales (Cnaf) des attentes des adolescents et des élus en matière d'animation urbaine et de loisirs ${ }^{1}$ montre que les enfants en sont globalement satisfaits jusqu'à l'âge de onze ans, mais qu'ils manifestent un fort désintérêt pour les structures de services une fois plus âgés (Cnaf, 2009a). Une étude menée auprès des collégiens d'une commune de l'agglomération bordelaise corrobore ce résultat (Zaffran, 2000) : il s'agit d'une enquête par questionnaire auprès de l'ensemble des collégiens de Floirac, une commune limitrophe de Bordeaux située sur la rive droite de la Garonne. L'échantillon de 800 individus environ se répartit entre un collège réputé populaire et difficile situé dans le bas de la commune et un collège situé dans le haut, recrutant beaucoup plus de catégories sociales favorisées. Calculé sur la totalité de l'échantillon, le taux de fréquentation des centres d'animation par les adolescents est très en dessous du taux attendu par les opérateurs municipaux : 29,7\% seulement s'y rendent de manière occasionnelle, et les

1. Conduite en 2007 et 2008, cette évaluation explore un ensemble de questionnements autour des attentes des familles, des jeunes, des élus et des partenaires, et cherche à identifier la plus-value apportée par les Caf, et à interroger les modalités d'intervention des accueils de loisirs des enfants et des jeunes âgés de six à dix-huit ans. Trois méthodes ont été utilisées pour investiguer ces points : 1. une synthèse des attentes des familles et des jeunes à partir d'études locales relayées par une quarantaine de Caf ; 2 . une enquête auprès d'élus de 300 communes représentatives, par questionnaire téléphonique, sur leur connaissance des problématiques territoriales, l'organisation de la compétence enfance-jeunesse, les grandes orientations, les effets perçus du contrat temps libre et du partenariat avec la Caf ; 3. huit monographies de territoires diversifiés en fonction de leur situation géographique, le niveau de structuration du partenariat, la configuration locale, des typologies différentes des familles (cf. Cnaf, 2009a, p. 2, encadré 2). autres s'en désintéressent. À l'instar de l'évaluation de la Cnaf, l'enquête pointe un effet de ciseau entre la montée en âge et la baisse de fréquentation, avec l'âge de douze ans comme point de rupture.

Sur le plan de l'analyse, la baisse d'intérêt à douze ans pour les équipements urbains pourrait s'expliquer par la faiblesse de l'offre et les freins en tout genre à leur accès. Or, qu'il s'agisse de l'agglomération bordelaise ou de l'ensemble des territoires évalués par la Cnaf, l'offre de loisirs est relativement bien répartie, et représente un investissement important des familles et des institutions (Cnaf, 2011, p. 35). Ce constat se confronte au problème sociologique de l'irréductibilité des conduites sociales au modèle économique qui détermine le choix des consommateurs en fonction d'une maximisation de son utilité. Les élus et les acteurs locaux font des efforts importants pour informer les adolescents des activités et des aides financières existantes. Les structures dédiées aux adolescents, pourtant dotées d'activités à la fois nombreuses et peu onéreuses, ne suscitent pas chez eux un intérêt à la hauteur des attentes des élus². Ce désintérêt ne laisse pas d'interroger ces derniers, voire de les inquiéter, d'autant que la plupart déclarent avoir une bonne connaissance des besoins des enfants, mais avouent pour $76 \%$ d'entre eux leur méconnaissance des attentes des adolescents. Ils sont aussi $38 \%$ à estimer que les jeunes représentent

2. Si l'article ne traite que des adolescents, un regard sur les attentes des parents permettrait de pointer les écarts entre ce que les premiers recherchent et ce que les seconds réclament. En outre, les attentes des familles à l'égard de leurs grands enfants sont « une vie scolaire "réussie", une vie sociale organisée et encadrée, une vie amicale autonome si possible supervisée par la famille» (Cnaf, 2009b, p. 30).

Les Annales de la recherche urbaine $\mathrm{n}^{\circ} 111$, 2016, pp. 68-77, (C)MEDDE, Puca. 
un problème d'incivilité, de conduites addictives et de désœuvrement (Cnaf, 2009b). L'expression de leur désarroi augmente devant l'inefficacité des intentions, sans doute bonnes, de proposer des services à la disposition des adolescents afin qu'ils puissent en tirer profit, et atteindre de la sorte l'objectif principal qu'ils assignent aux équipements : " l'accès à la citoyenneté par un comportement sociable »(Crépin, 2008). Mais plus largement, la baisse de fréquentation des équipements soulève une interrogation d'ordre sécuritaire : où sont les adolescents et que font-ils quand ils ne sont ni dans les lieux d'encadrement urbain ni dans les lieux privatifs? Elle pose aussi une question d'ordre sociologique : pourquoi les adolescents ne vont-ils plus dans les endroits qu'ils appréciaient jusque-là ?

Cette seconde interrogation invite à bâtir une explication qui ne fasse appel ni à des éléments psychophysiologiques (trop réducteurs pour faire l'objet d'une théorisation sociologique), ni à l'irrationalité des conduites adolescentes (largement préjugée par le sens commun). Elle suppose au contraire de considérer les adolescents comme des sujets à part entière, dont l'action est socialement orientée vers un but (Cuin, 2011). On s'autorise de la sorte à traiter la défection comme une forme de non-recours, c'est-à-dire une intentionnalité réfléchie dans un contexte de dotations économiques et d'informations suffisantes (Warin, 2012). Plus avant, si l'explication à tirer du «problème » de la fréquentation des équipements urbains présente un intérêt sociologique, elle peut (et doit) être utile aux élus dans le cadre d'une politique de l'adolescence qui prenne en compte les usages de la ville par les adolescents et les préoccupations des édiles en matière de gestion urbaine des flux des personnes. Ces objectifs organisent l'article en trois parties. La première partie aborde la notion d'adolescence sous un angle sociologique afin de replacer cet âge de la vie dans le cadre normatif de la ville, et plus largement de notre société. La seconde partie explique les raisons de leur retrait des équipements à partir des épreuves que les adolescents sont tenus de franchir, et que la mobilité aide à franchir. La dernière partie esquisse une politique de l'adolescence libérée du biais cognitif qui empêche de voir la ville comme un lieu d'expérimentation et l'adolescence comme l'épreuve de l'autonomisation.

\section{Regard sociologique sur l'adolescence}

Vue sous un angle sociologique, l'adolescence est le travail accompli par l'enfant pour être soi parmi les autres (Singly de, 2004). Ce travail, qui illustre une partie de ce que la sociologie range sous le concept de modernité avancée, se manifeste dans les efforts pour quitter l'enfance par l'affirmation d'un soi individualisé qui cultive son originalité et accroît son autonomie sociale. La maîtrise progressive du monde par soi-même, l'autonomie donc, s'adosse aux règles tantôt imposées par autrui, tantôt reconstruites par soi-même, mais négociées à plusieurs. De ce fait, la période de l'adolescence est caractérisée par une recherche de liberté et d'authenticité, ce qui suppose un travail de détachement des figures tutélaires de l'enfance et une sortie progressive et par alternance des cadres de la socialisation verticale 3 .

$\mathrm{Si}$ cette autonomie transite par tous les domaines d'activités (l'école, la famille, les loisirs), elle butte sur une police de la ville qui s'alimente de peurs paniques (Oblet, 2008). L'urbanité est réduite à la gestion des risques et à un traitement pragmatique de la jeunesse par un encadrement accru. En cela, la ville est pensée contre les adolescents plutôt qu'avec eux. Du reste, cet encadrement n'est pas nouveau. Par le passé, les adolescents transitaient par des « lieux d'institution » - l'école, l'église, l'usine qui étaient des pôles de socialisation (Thiercé, 1999). La jeunesse représentait un enjeu pour ces réseaux qui exerçaient leur influence dans des lieux d'encadrement relevant des patronages laïques et religieux. Ces associations contribuaient au maillage idéologique du territoire et assuraient une socialisation continue des jeunes. Les mouvements de jeunesse prolongeaient ainsi l'église, l'école ou l'usine, et assuraient de la sorte une formation totale des adolescents socialisés.

Le déclin des institutions au milieu du $\mathrm{XX}^{\mathrm{e}}$ siècle marque une évolution des lieux d'institution dans le sens d'une anthropologie nouvelle qui valorise l'individu authentique (Ehrenberg, 1995; Dubet, 2002). Les institutions ne s'organisent plus autour d'un programme institutionnel qui définit clairement l'action des professionnels et le rôle des individus. Les individus sont sommés de trouver par eux-mêmes les leviers de leur formation tout en veillant à défendre leurs intérêts propres. C'est pourquoi l'école gère aujourd'hui des tensions dues à la compétition scolaire et aux demandes de reconnaissance de la culture adolescente des élèves (Barrère, 2011); que les églises se vident de leurs fidèles, qui, sans se détourner nécessairement de la religion, se fient à leur bricolage religieux plutôt qu'à un dogme (Hervieu-Léger, 1999) ; enfin que les grandes fédérations de jeunesse peinent à s'orienter par les valeurs qui incarnent leurs mouvements originels, et courent le risque de sacrifier les vertus éducatives sur l'autel du marché (Poujol, 2000). Les adolescents grandissent avec la certitude qu'il faut s'individualiser, mais dans un contexte où les références sont moins stables que par le passé. En conséquence, ils sont tenus de «lacer » par eux-mêmes les fils de leur individualité, et ce faisant sont confrontés à l'épreuve de la mobilité.

3. C'est d'ailleurs un principe sur lequel les élus insistent, puisque dans l'enquête Cnaf citée, ils déclarent attendre des jeunes qu'ils deviennent autonomes. 


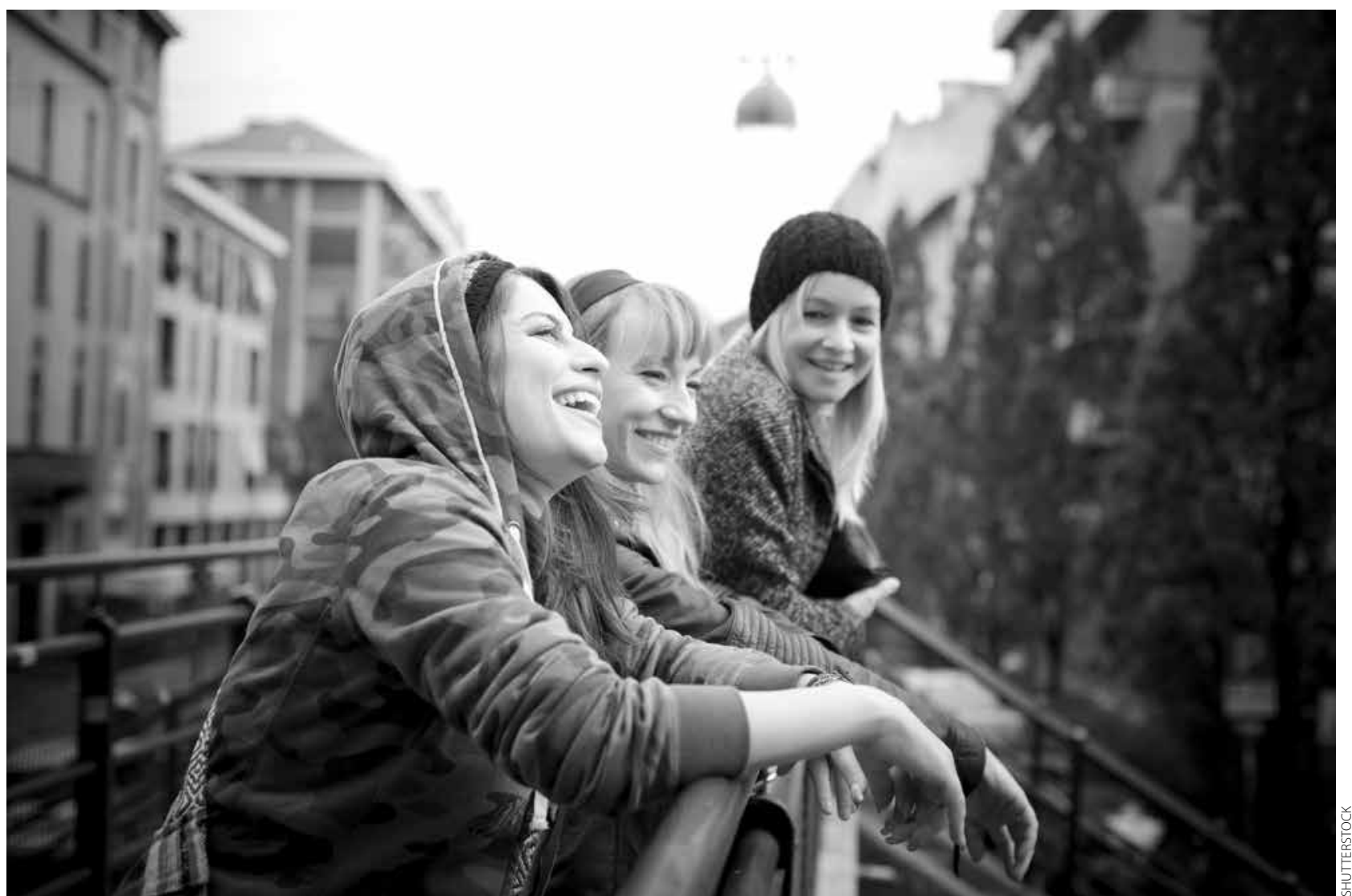

\section{La ville support d'une autonomie élargie?}

Etre soi est une injonction normative forte, constitutive de l'expérience sociale des adolescents. Or, ces derniers construisent une grande part de leur expérience dans des lieux formels et délimités : la maison, l'école, les structures sportives et de loisirs, etc. L'extension des domaines de l'autonomie à laquelle ils aspirent, par nécessité et par choix, butte sur la tutelle des adultes qu'ils croisent à l'école ou dans leur environnement familial. Quitter l'enfance et entrer dans l'adolescence suppose alors de trouver et négocier des marges d'autonomie plus nombreuses afin d'investir progressivement de nouveaux espaces et de maîtriser un réseau de sociabilité propre (Metton-Gayon, 2009). Ce processus se caractérise par des goûts et des préférences élaborés dans le cadre d'une socialisation horizontale qui échappe en grande partie aux parents (Pasquier, 2005), et s'appuie sur une culture alternative composée de codes différents de ceux provenant des agents de socialisation primaire (Glévarec, 2002). Pour l'enfant, devenir adolescent consiste à utiliser les nouveaux droits que les adultes lui attribuent ou qu'il conquiert pour les expérimenter dans des lieux ouverts, et de la sorte à accroître son autonomie sociale. L'adolescence est l'épreuve de l'autonomie par la capacité, acquise ou conquise, d'élaborer individuellement et collectivement un monde à soi hors des lieux imposés de l'enfance.
La ville permet de franchir cette épreuve. Axée sur le thème des loisirs et du temps libre, une enquête par entretiens, réalisée auprès de collégiens et collégiennes résidant dans l'hypercentre gentrifié de Bordeaux et dans des quartiers populaires et excentrés du centre-ville, montre que les territoires urbains sont un support commun qui, par les usages qu'ils en font et le rapport qu'ils en ont, peuvent favoriser le passage de l'enfance à l'adolescence (Zaffran, 2010). La découverte puis l'occupation rituelle des espaces urbains permettent de s'éprouver à travers les liens d'appartenance qui naissent et s'affirment lors des mises en scène de soi avec les autres dans des lieux aisément repérables et à des moments précis (Félonneau, 1994). Au sortir d'une enfance fortement dépendante des adultes, les adolescents sont irrésistiblement attirés vers des endroits qui les éloignent progressivement des lieux de la socialisation primaire, et les conduisent pas à pas sur des terrains d'expériences propres à assouvir des aspirations que la famille et l'école ne sont pas ou ne sont plus en mesure de satisfaire. Passer socialement de l'enfance à l'adolescence consiste donc à faire du territoire un support d'individualisation. En l'espèce, la ville en est le cadre idéal, précisément par les pôles d'attraction qu'elle recèle, les lieux d'ancrage qu'elle offre et les mobilités qu'elle occasionne.

Ces mobilités suivent un parcours dicté à la fois par des conditions objectives et la perception des lieux comme un 
terrain de consommations, d'échanges, ou de risques. Sur ce plan, des différences existent entre les garçons et les filles : les premiers ont tendance à fréquenter les laser-game ou les bowlings, les secondes préfèrent les cinémas et les lieux plus " culturels ». Elles préfèrent aussi se déplacer d'un lieu à un autre, et ne restent pas longtemps au même endroit. En d'autres termes, elles « tracent », pour reprendre leur expression, et se faisant « traceuses » rendent leur présence sinon moins visible du moins plus " labile». Le mouvement devient une ressource car il donne l'impression qu'elles se rendent à un endroit précis ou qu'elles sont attendues (Buffet, 2005). Mais tous, garçons et filles, s'accordent à dire que ces lieux sont l'occasion, lorsque les parents les autorisent à s'y rendre, de se retrouver entre pairs. Le groupe arpente les rues piétonnières pour la concentration d'offres commerciales variées et attractives qu'elles proposent, et les sorties suivent souvent un circuit

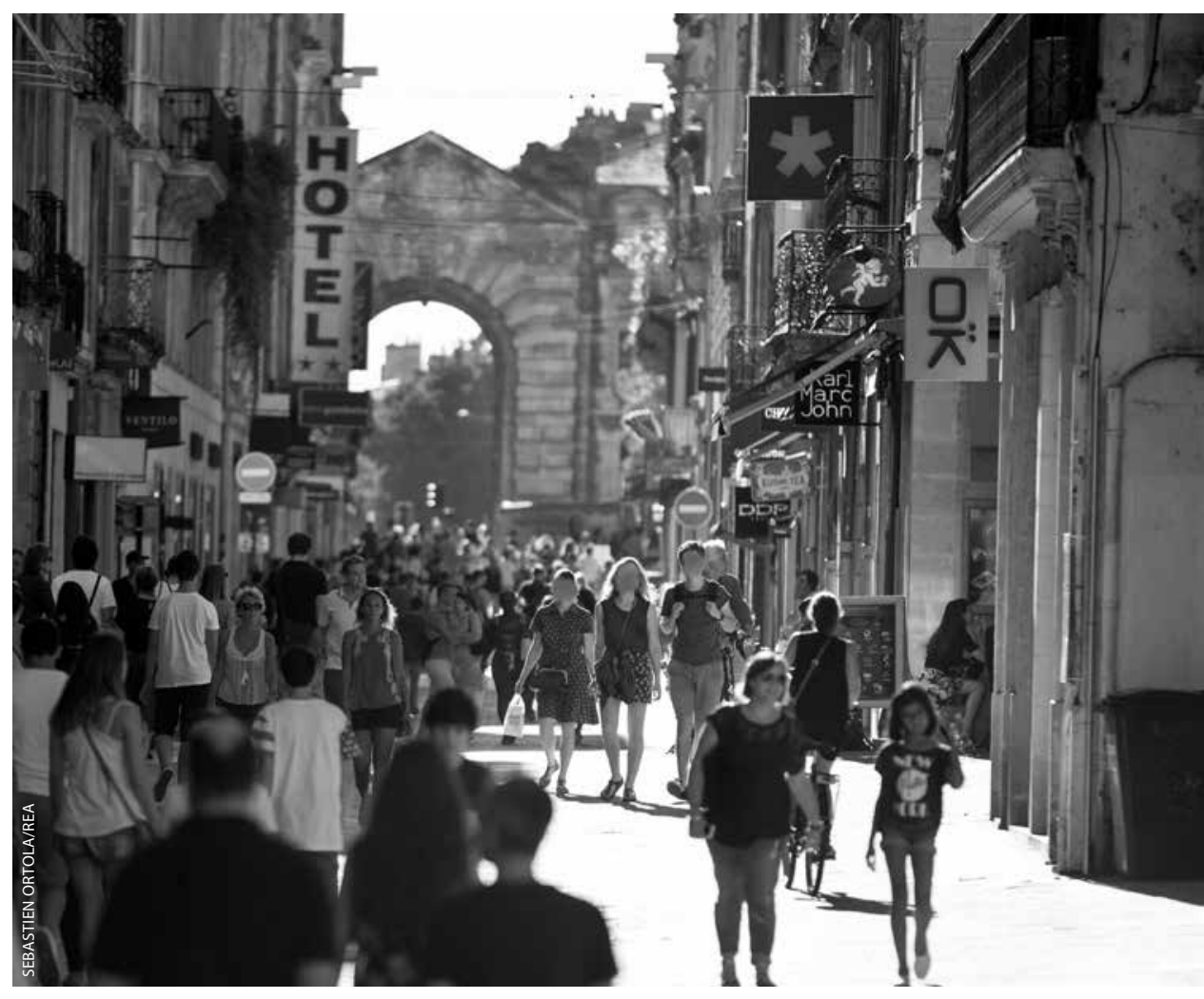
balisé par des lieux de passage (un établissement de restauration rapide, une salle de cinéma, etc.), et des lieux ouverts dans lesquels il se consacre à des petits riens jusqu'à l'heure du retour. Ce circuit se fait dans un entre-soi d'autant plus nécessaire qu'ils perçoivent la rue comme un lieu inquiétant du fait de l'absence des " filtres » du quartier auxquels ils sont accoutumés et qui leur garantissent une tranquillité relative : «On n’est pas dans notre élément, je trouve ! Je ne sais pas, mais la rue, ce n'est pas ce que je préfère... Je suis moins à l'aise dans la rue qu'autre part " (Lorie, adolescente de 13 ans d'origine sociale favorisée qui habite dans le centre-ville de Bordeaux). Cette image n'échappe pas à la conscience des garçons qui sont nombreux à souligner que la nuit tombée, la rue prend un visage encore plus menaçant. Cette crainte les pousse à emprunter les grands axes pour se rendre dans des lieux précis, sans chercher à s'approprier véritablement l'espace public. Comme les filles, ils sont usagers d'une ville qu'ils sollicitent en tant que consommateurs. Le reste du temps, ils préfèrent leur quartier pour la sécurité que les routines urbaines leur apportent.

Ce rapport à l'espace et l'image qu'il renvoie concernent surtout les adolescents proches du centre-ville. Ceux qui en sont éloignés ${ }^{4}$ trouvent regrettable qu'il détienne le monopole de l'animation. La distance qui les sépare des lieux d'animation transforme chaque sortie en expédition.

4. Ils ont pour point commun d'habiter des quartiers éloignés du centre-ville et d'appartenir à une classe populaire.

Cela suppose en effet un minimum d'organisation, et de supporter des coûts qui sont moins économiques que temporels : l'accès au centre-ville nécessite d'utiliser plusieurs lignes de transport en commun et demande un temps de trajet important. Bouger se paie au prix du temps, ce qui oblige à des arbitrages. La mobilité se restreint et les activités auxquelles se livrent les adolescents sont choisies en fonction de la proximité au quartier :

«Enquêteur : Cela vous pose-t-il un problème que la plupart des activités soient au centre-ville de Bordeaux?

Alexis ${ }^{5}$ : Oui parce qu'il faut quand même se déplacer, il faut se décider à prendre le bus.

Sofian : Comme c'est en ville, on se dit : "Non, c'est super loin! " En plus il faut prendre le bus, et d'ici il faut en prendre deux, donc c'est long, même si on prend le tram. »

De ce point de vue, la mobilité des adolescents dépend de paramètres identiques à d'autres catégories de personnes plus âgées ${ }^{6}$ : l'éloignement du lieu de résidence, l'offre de transports et la combinaison des modes de déplacement, les dépendances multiformes (économiques, familiales), les compétences individuelles comprenant la connaissance des réseaux de transport et la maîtrise des connexions. Cependant, la différence avec les autres classes d'âge réside

5. Alexis et Sofian ont quatorze ans et habitent un quartier populaire réputé sensible de Bordeaux.

6. Voir à ce propos l'analyse de Choplin et Delage (2011) sur les étudiants de l'Est francilien. 
dans la gestion conjointe de l'agenda familial et scolaire. Sofian et Alexis font en effet la démonstration que la gratuité du titre de transport ou la détention d'une carte d'abonnement avantageuse (on pense à l'exemple francilien de la carte Imagine R) ne suffisent pas à compenser une dimension constitutive de la mobilité des adolescents, en l'occurrence le temps dégagé des contraintes scolaire et familiale, sa représentation et son utilisation.

Lorsqu'ils ne peuvent réunir les conditions pour profiter pleinement de la ville ou que les coûts de la mobilité sont trop importants, les adolescents trouvent à proximité de leur domicile les lieux d'autonomisation ${ }^{7}$ qu'ils aménagent dans l'idée de s'y retrouver, voire de s'y installer, convertissant ainsi les entraves à la mobilité en ressources territoriales. En somme, quand les adolescents ne peuvent faire de la mobilité un moyen de briser la chaîne qui les retient aux territoires imposés (Kokoreff, 1993), ils accommodent les lieux de fixation par une redistribution des fonctions qui leur sont dévolues. Ils recomposent l'espace pour y "faire salon » (Lefrançois, 2006). Leur expérience urbaine et sociale s'élabore ainsi depuis le lieu de résidence, et reste par conséquent fortement dépendante des rapports qu'ils entretiennent à l'espace local (Oppenchaim, 2011). Les manières d'habiter le quartier font du contrôle de son accès un enjeu de pouvoir, en particulier pour les bandes de garçons qui le transforment facilement en " arène identitaire ${ }^{8}$ » :

«Enquêteur : Vous fréquentez souvent ce skatepark?

Jean : Non, pas souvent! Quand nous, on y va, tous les Aubiers nous attendent devant, et on se bagarre tout le temps.

Enquêteur : Tu veux dire qu'ils sont là-bas, et ils ne vous laissent pas approcher?

Jean : Ouais.

Enquêteur : Et d'après vous, ça appartient plus aux Aubiers qu'à Bacalan ou...

Joris : Normal ! C'est dans leur quartier, il y a plein de cités là... Ils s'y sont mis tous... On essaye un peu de rentrer dedans, mais ils se mettent en face et il n'y a qu'une chose à faire, c'est se battre! Ils ne savent pas faire autre chose ! Parler, ils ne savent pas! Alors on se bagarre. »

Les filles n'ont pas leur place dans les affrontements. Cela exige une virilisation qu'elles ne parviennent pas à assumer complètement, à moins de «faire comme les garçons ».

7. C'est l'autre différence avec les jeunes plus âgés qui tentent individuellement de développer des stratégies leur permettant de gagner en mobilité et par extension en autonomie (Choplin et Delage, 2011). Les adolescents sont quant à eux moins dans une logique stratégique d'extension du territoire lato sensu que d'investissement de leur territoire stricto sensu.

8. Âgés de treize ans environ, ils habitent le quartier populaire de Bacalan situé au nord de Bordeaux et au bord de la Garonne, coincé entre les bassins à flots au sud, le pont d'Aquitaine au nord et à la fin des boulevards à l'ouest ; l'arrivée du tramway a désenclavé ce quartier populaire.
Elles optent plutôt pour un rôle de modératrices, de manière à désamorcer le conflit mais aussi à tenir leur rang : « À un moment, il y en avait un du Grand Parc qui voulait taper un gars de Bacalan, et il y a eu une embrouille... Et en fait, il y a un mec qui est super connu au Grand Parc qui a aidé pour pas que ça se passe et il s'est mis entre les deux, il lui a fait: "Qu'est-ce que tu fais ? Tu n'as pas à venir ici taper quelqu'un du Grand Parc, ici ce n'est pas chez toi, alors tu retournes chez toi !" Et moi j’étais là, j’étais juste à côté parce que s'il y avait eu une bagarre je me serais mise au milieu, parce que des fois, quand on les connaît bien, on sait qu'ils nous écoutent et si on leur dit d'arrêter, ils arrêteront. Donc on reste là. » (Inès, 14 ans, habite le quartier du Grand Parc situé au nord de Bordeaux).

Lorsqu'ils disposent des ressources suffisantes pour se déplacer, les adolescents excentrés du centre-ville utilisent la ville comme un marché de biens et de services, mais s'y promènent aussi sans objectif précis, pour passer le temps. L'éloignement et la mobilité qu'elle suppose permettent d'avoir un regard plus étendu sur la ville que dans le cas des adolescents proches du centre-ville, et les dotent d'une connaissance nettement plus pratique de la ville. Ce sont moins les lieux qui ont leur importance que le fait de ne pas être là où ils devraient être ou là où ils sont attendus. Ils s'approprient la ville par une exploration et des expérimentations singulières qu'autorisent les circuits plus ouverts, occasionnant de multiples déplacements et laissant une grande part à l'« aventure » partagée avec le groupe de pairs. Peu importe alors le lieu puisque c'est l'esprit du groupe qui confère à l'espace son sens et sa fonction (Simmel, 1999) : « On se donne rendez-vous avec des copains et on fait un tour dans Saint-Pierre?. Ensuite, l'après-midi, on va toujours en ville parce qu'on voit du monde dans la rue, et à des moments on s'arrête et on s'assoit un peu. On reste par exemple place Saint-Projet, on discute et on attend que le temps passe » (Nolan, 13 ans, habite le Grand Parc, un ensemble urbain situé à proximité du quartier gentrifié des Chartrons).

Ces manières individuelles et collectives de prendre possession de l'espace superposent la ville des adolescents à la ville des adultes. La ville des uns est d'autant plus différente de la ville attendue et voulue par les autres que d'une part ils la hissent à leur échelle et l'investissent à leur mesure, et d'autre part, ils l'élargissent au fur et à mesure qu'ils avancent en âge. Il va de soi que cette expérience urbaine est marquée par les appartenances ou les différences sociales et que la mobilité est dépendante d'un ensemble de facteurs, mais tous la voient comme une réponse aux lieux d'encadrement qui comblaient plus ou moins leurs besoins lorsqu'ils étaient enfants, mais qui sont dénués de sens à l'adolescence. Cette quête de sens se déroule au-dehors, dans la ville, qui permet la mobilité entre les espaces, et

9. Un quartier gentrifié de l'hypercentre de Bordeaux. 


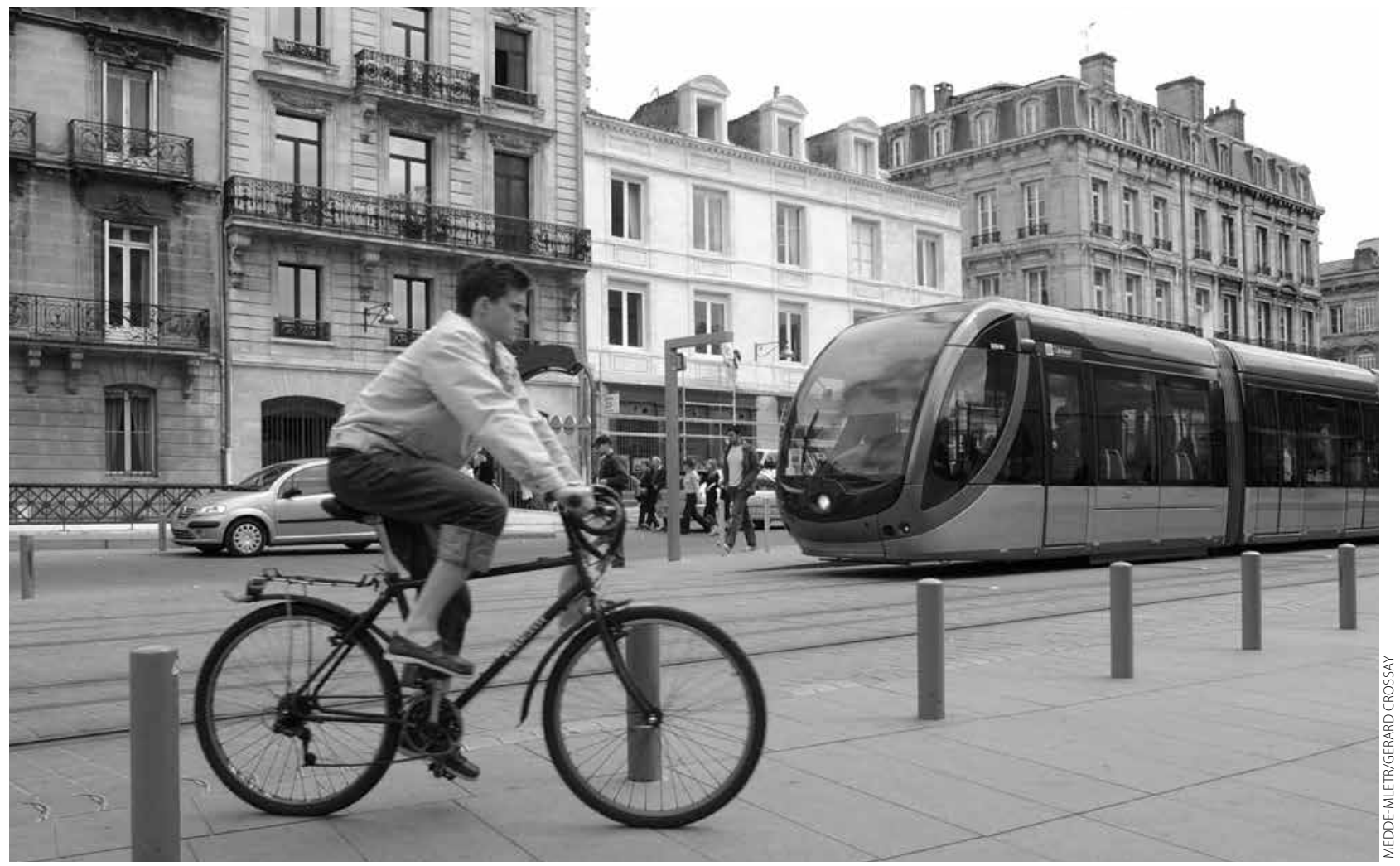

par conséquent l'affirmation identitaire et l'expérience de l'autre (Grafmeyer et Authier, 2008). Le concept de mobilité répond dans ce cas moins aux dynamiques urbaines contemporaines (Bassand, Kaufmann et Joye, 2007) qu'à la construction de soi à l'adolescence. De sorte que la mobilité des adolescents est l'ensemble des moyens et des compétences qui favorisent le déplacement et l'accès à des lieux, les déambulations entre les ancrages urbains durables et temporaires ayant surtout une visée identitaire.

\section{Une politique urbaine de l'adolescence}

Si la mobilité adolescente a des incidences sur l'urbain, il importe aussi de saisir l'enjeu des dispositions subjectives et objectives du changement d'espace-temps, afin que les élus répondent à une réaction adolescente de nature plus identitaire qu'instrumentale. Pour cela, la mobilité des adolescents ne peut pas se concevoir comme un déplacement fortement balisé par les institutions régulatrices des conduites sociales, mais plutôt comme un cheminement moins prévisible car plus conjoncturel (Bourdin, 2005). Or, les déplacements constitutifs d'une mobilité libérée pour un temps des régulations verticales et affranchie des lieux d'encadrement ne cessent d'interroger les élus, tenus de gérer la tension entre un désir de satisfaire les besoins des adolescents et un souci de prévention des risques dans l'espace public (Oblet, 2008). Leur inquiétude devant le faible taux de fréquentation des équipements qui leur sont dédiés par les adolescents justifie à leurs yeux le besoin d'évaluer le fonctionnement des structures et les pratiques professionnelles. Dépendantes d'une vision de l'espace public comme un lieu de répartition ordonnée des personnes et des flux, ces évaluations sont adossées au modèle du loisir comme un marché sur lequel il suffit de trouver un point d'équilibre entre les besoins des adolescents et une offre publique de services ajustée. De cette manière, elles confortent la conviction que l'adolescence mérite un accompagnement éducatif par les adultes dans un cadre fermé. Il s'agit donc de consulter les adolescents en vue d'établir la liste de leurs besoins en termes d'offre de loisirs. Or, la défection des équipements urbains est, comme on vient de le voir, une réponse des adolescents au besoin d'expérimentation de la ville différente de celles vécues (et donc éculées) dans les lieux d'encadrement, et que la mobilité favorise.

Une politique urbaine de l'adolescence doit donc défendre les lieux d'expérimentation identitaire dans une ville "délacée », puis l'assumer, puisque cette expérimentation interroge la place de chacun dans une ville ouverte à tous les publics et autorisant un partage commun de l'espace public. Àcetégard, les demandes des adolescents en matière de loisirs sont assez précises dans l'enquête Cnaf. Ils réclament une participation à la vie sociale, la création de liens intergénérationnels, l'implication active dans des projets attractifs à leurs yeux. Ils expriment par ailleurs un besoin d'expériences collectives ainsi qu'une demande d'encadrement qui, selon leurs termes, prendrait la forme d'un « encadrement invisible » au contenu assez précis : 
pouvoir faire des choses en étant accompagné par un adulte qui, sans être interventionniste, serait proche d'eux en termes de goûts et de comportements, qui assurerait une fonction d'aide et de conseil, qui serait apte à maîtriser les débordements éventuels. Cet encadrement invisible prendrait du relief dans les lieux ouverts que les adolescents privilégient. Toujours dans cette enquête, à la question « lorsque tu restes avec tes copains ou tes copines en semaine après les cours, où allez-vous ? ", les adolescents répondent principalement : « rester dans la rue » $32 \%$ souvent et $42 \%$ de temps en temps), " aller chez un copain » (16\% souvent et $55 \%$ de temps en temps), «aller dans un café » (19\% souvent, $40 \%$ de temps en temps), «aller chez soi » (9\% souvent, $44 \%$ de temps en temps).

Leur perception de la ville se structure davantage par les rues qui les émancipent que par les sites qui les ramènent inexorablement au statut d'enfant ou d'élève. La ville est vécue comme un lieu de fantasmes, un terrain d'aventures, un territoire de rencontres, un espace ouvert aux autres et sur les autres, mais vidé de la présence des adultes. Elle les attire par la possibilité de créer et trouver des lieux fermés dans des espaces ouverts. Pour ces raisons, une politique urbaine de l'adolescence doitfaciliter la mobilité des adolescents (des zones urbaines et rurales) vers la ville et dans la ville. La propension des adolescents à bouger et partir de la sorte à la découverte de nouveaux mondes sociaux colore la socialisation urbaine d'une mobilité qui s'apparente à une aventure temporaire et transitoire. Peu importe en effet la distance parcourue ou la destination recherchée, puisque le cheminement compte plus que la destination et que, comme l'indique Stefan Zweig dans Le monde d'hier, "les chemins empruntés ramènent toujours à soi ». La mobilité est une parenthèse plus ou moins brève entre deux états stables; sitôt introduite, elle ouvre une brèche dans le quotidien (Barrère et Martuccelli, 2005). Elle comporte aussi des risques en obligeant l'adolescent à sortir des lieux d'assignation qui enferment et protègent à la fois (Lapeyronnie, 2008) : la mobilité recèle ainsi une part d'aventure, l'entrainant par des chemins et dans des lieux moins rassurants que ceux qui lui sont familiers.

La transition entre l'enfance et l'adolescence s'accompagne d'une baisse importante de la fréquentation des lieux d'encadrement ${ }^{10}$. Quand ils avancent en âge, les adolescents ouvrent des fenêtres sur la ville et referment derrière eux les portes des équipements urbains afin de se ménager des espaces à eux. Mais ce rejet des équipements urbains est moins une critique adressée aux contenus de l'offre de loisirs que la manifestation tangible d'une

10. Ce désintérêt oblige à des expérimentations comme le centre de loisirs de rue, le rollerpark, les sorties culturelles ou sportives, etc., qui d'ailleurs sont autant de propositions faites par les adolescents pour «sortir des lieux formels » (Cnaf, 2009b, p. 45). récusation, originale à cet âge de la vie, des normes d'une société dans laquelle leur place est réduite à des lieux dont la fonction est d'éduquer, de distraire et d'encadrer. Le contournement volontaire des équipements fait des adolescents des acteurs à part entière, c'est-à-dire des individus qui, au sortir de l'enfance, élargissent leur capacité d'action et usent, avec force et habileté, de ressources critiques pour ménager dans la ville des îlots de subjectivation. Ils creusent leur trou et bâtissent une ville à eux, moins dans le détachement d'une introspection solitaire qu'en recomposant les relations sociales et en contournant collectivement les lieux d'encadrement. En conséquence, faire défection est un des actes fondateurs de la revendication à l'adolescence : choisir par soi-même ses activités et ses déplacements.

Si l'on traite l'action publique avec les cadres cognitifs et normatifs qui en constituent le cœur, et qui mélangent des éléments de connaissance, des idées, des représentations ou des croyances sociales (Muller, 2000), les modes d'intervention vis-à-vis des adolescents resteront peu opérants tant que l'appréhension de l'adolescence dans la ville ne se libèrera pas de l'idée que l'absence d'encadrement institutionnel est un danger pour l'adolescent et un risque pour autrui. Plutôt que de restreindre les lieux d'autonomisation sociale des adolescents, la conciliation des contraintes des usages de l'espace urbain avec les attentes de tous les utilisateurs doit prendre au sérieux les raisons pour lesquelles les adolescents se détournent des lieux de socialisation contrainte, sans que ce rejet n'apparaisse comme irrationnel ou anomique.

Au final, l'enjeu est à la fois politique et éducatif, car il s'agit de donner aux adolescents l'encadrement invisible qu'ils réclament. Celui-ci traduit un désir de détachement plus qu'une volonté de rupture avec les figures tutélaires, mais aussi une volonté de prendre place dans une ville et dans des lieux qui les attirent autant qu'ils les inquiètent. Ces lieux sont fatalement changeants. Ils contribuent aussi à faire de la mobilité un principe d'une expérience originale par l'appel à la cognition de l'espace et du temps (Le Breton, 2005). La mobilité suppose en effet une représentation des lieux et des territoires que l'expérience pratique de la ville aide à élaborer. L'exploration de la ville permet de poser les jalons cognitifs d'un espace dont l'appropriation est facilitée par la capacité grandissante à se le représenter mentalement. La mobilité implique aussi une maîtrise du temps par les découpages temporels à faire autour du trajet, des activités et des obligations. Cette manière de saisir l'espace et le temps les amène à se réfugier dans des contre-espaces à distance physique et symbolique des lieux formels (l'école, les équipements urbains, etc.) bâtis et aménagés pour eux ${ }^{11}$. Elle les aide tout simplement à grandir.

11. Sur ce point, voir M. Foucault (1994). 


\section{Références bibliographiques}

Barrère A., Martuccelli D., (2005), « La modernité et l'imaginaire de la mobilité : I'inflexion contemporaine », Cahiers internationaux de sociologie, vol. CXVIII, pp. 55-79.

Barrère A., (2011), Léducation buissonnière: quand les ados se forgent par eux-mêmes, Paris, Armand Colin.

Berger P., Luckmann T., (1989), La construction sociale de la réalité, Paris, Méridiens Klincksieck.

Bourdin A., (2005), «Les mobilités dans le programme de la sociologie », Cahiers internationaux de sociologie, CXVIII, janvier-juin, pp. 5-21.

Buffet L., (2005), «Les différences sexuées de l'accès à l'espace urbain chez les adolescentes de banlieues populaires », in Buisson M.-A. et Mignot D. (dir.), Concentration économique et ségrégation spatiale, Bruxelles, De Boeck.

Choplin A., Delage M., (2011), « Mobilités et espaces de vie des étudiants de l'Est francilien : des proximités et dépendances à négocier », Cybergeo: European Journal of Geography, [en ligne], http://cybergeo. revues.org/23840, document 544, (mis en ligne le

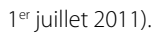

Cnaf, (2009a), « La contractualisation avec les Caf : quelle contribution à la construction collective d'une politique enfance jeunesse? », Essentiel, n²81, janvier.

Cnaf, (2009b), «Évaluation de la politique de l'enfance et de la jeunesse des Caf. Attentes des familles et des jeunes. Attentes des élus. Territoires », Dossier détude, $n^{\circ} 113$, février.

Cnaf, (2011), « Les relations entre les parents et leurs enfants à l'aune des loisirs des adolescents », Dossier d'études, n 140, mai.

Crépin C., (2008), « La politique temps libre, enfance et jeunesse : un projet national d'évaluation de politiques locales », Informations sociales, $\mathrm{n}^{\circ} \mathrm{6}$, pp. 150-159.
Cuin C.-H., (2011), « Esquisse d'une théorie sociologique de l'adolescence », Revue européenne des sciences sociales, 2, pp. 49-72.

Dubet F., (2002), Le déclin de l'institution, Paris, Seuil.

Ehrenberg A., (1995), Lindividu incertain, Paris, Hachette.

Félonneau M.-L., (1994), « Les étudiants et leurs territoires. La cartographie cognitive comme instrument de mesure de l'appropriation spatiale », Revue française de sociologie, 35-4, pp. 533-559.

Foucault M., (1994), « Des espaces autres. Hétérotopies », in Dits et écrits, t. IV, 1980-1988, Paris, Gallimard, pp. 752-762.

Galland O., (1991), Sociologie de la jeunesse. L'entrée dans la vie, Paris, Armand Colin (Collection U).

Giddens A.s (1987 [1984]), La constitution de la société, Paris, Puf.

Grafmeyer Y., Authier J.-Y., (2008), Sociologie urbaine, Paris, Armand Colin.

Hervieu-Léger D., (1999), Le Pèlerin et le Converti : la religion en mouvement, Paris, Flammarion.

Kokoreff M., (1993), « L'espace des jeunes. Territoires, identités et mobilité ", Annales de la recherche urbaine, $n^{\circ}$ 59-60, juin-septembre.

Lapeyronnie D., (2008), Ghetto urbain. Ségrégation, violence, pauvreté en France aujourd'hui, Paris, Robert Laffont.

Le Breton E., (2005), Bouger pour s'en sortir. Mobilité quotidienne et intégration sociale, Paris, Armand Colin.

Lefrançois D., (2006), Le parking dans le grand ensemble. Entre "habiter, circuler, travailler, se recréer», thèse de doctorat, université Paris XII/Institut d'urbanisme de Paris.
Metton-Gayon C., (2009), Les adolescents, leur téléphone et Internet. «Tu viens sur MSN? », Paris, L'Harmattan.

Muller P., (2000), « L'analyse cognitive des politiques publiques : vers une sociologie politique de l'action publique », Revue française de science politique, v. 50, n², pp. 189-208.

Oblet T., (2008), Défendre la ville. La police, l'urbanisme et les habitants, Paris, Puf.

Oppenchaim N., (2011), Mobilité quotidienne, socialisation et ségrégation: une analyse à partir des manières d'habiter des adolescents de zones urbaines sensibles, thèse de doctorat, université Paris Est.

Poujol G. (dir.), (2000), Éducation populaire: le tournant des années soixante-dix, Paris, L'Harmattan.

Rayou P., (2006), « Ni tout à fait mêmes, ni tout à fait autres. Les territoires scolaires des enfants et des jeunes », in Sirota R. (dir), (2006), Éléments pour une sociologie de l'enfance, Rennes, Pur.

Simmel G., (1999 [1908]), Sociologie. Étude sur les formes de la socialisation, Paris, Puf.

Singly de F., (2004), Enfants adultes, vers une égalité de statuts, Paris, Éd. Universalis.

Thiercé A., (1999), Histoire de l'adolescence (1850-1914), Paris, Belin.

Warin P., (2012), « Le non-recours aux droits », Sociologies, [en ligne] http://sociologies.revues. org/4103, (mis en ligne le 15 novembre 2012, consulté le 4 mars 2014).

Zaffran J., (2000), Les collégiens, lécole et temps libre, Paris, La Découverte.

Zaffran J., (2010), Le temps de l'adolescence. Entre contrainte et liberté, Rennes, Pur. 
Biographie

JOËL ZAFFRAN est professeur de sociologie à

I'université de Bordeaux et chercheur rattaché au centre Émile Durkheim (CED UMR-CNRS 5116).

Ses travaux sur l'adolescence portent sur les

transitions d'âge et les temps sociaux qu'il aborde sous un angle sociologique. II a notamment publié «Déprise et emprise : les deux faces de l'épreuve de l'adolescence » in Ertul S. et al. (dir.), Subjectivation et redéfinition identitaire, Rennes, Pur, 2014 ; ainsi que « La chambre des adolescent(e)s : espace intermédiaire et temps transitionnel », Strenæ, 7, 2014, [en ligne] http://strenae.revues.org/1261.

joel.zaffran@u-bordeaux.fr 